\title{
CAN LITIGATION IMPROVE THE LAW WITHOUT THE HELP OF JUDGES?
}

\author{
ROBERT COOTER* and LEWIS KORNHAUSER**
}

$I_{s}$

the common law "efficient"? Do actual tort liability rules minimize the sum of accident costs and accident-prevention costs? Do actual nuisance rules lead to optimal levels of output, optimal location patterns, and optimal use of "clean" technologies? At the least, have common law rules improved (on an efficiency scale) over time? These questions have been raised explicitly and implicitly by the growing literature on law and economics. ${ }^{1}$

Three justifications for the statement that the common law tends towards efficiency have been offered: (i) judges actively seek efficiency; ${ }^{2}$ (ii) inefficient rules are litigated more often than efficient rules (differential litigation); ${ }^{3}$ and (iii) litigants who benefit from an efficient rule invest more in the litigation than those who favor an inefficient one. ${ }^{4}$ The first justification relies upon judicial insight to improve the law, whereas the second and third justifications do not.

Certainly the law can improve over time due to judicial insight. For example, it can be shown that the standard of negligence in liability law would evolve towards efficiency if judges amend it according to a simple calculation of benefits and costs. ${ }^{5}$ However, it is difficult to contend that

* Assistant Professor of Economics, University of California at Berkeley

** Assistant Professor of Law, New York University School of Law

The authors would like to thank the participants in the Sloan Seminar on Regulation at New York University, the participants in the informal seminar on law and economics at the Harvard Law School, and an anonymous referee for helpful comments.

${ }^{1}$ Richard A. Posner, Economic Analysis of Law (1st ed. 1973) at 320-28, is an early, explicit claim that the common law process is superior to the legislative process in attaining efficiency. The first four chapters of the book argue for the proposition that a variety of common law rules are in fact efficient.

2 Richard A. Posner, Economic Analysis of Law 399-418 (2d ed 1977). Posner may be arguing that no other motivational assumption is plausible.

${ }^{3}$ Paul H. Rubin, Why Is the Common Law Efficient?, 6 J. Legal Stud. 51 (1977). George L. Priest, The Common Law Process and the Selection of Efficient Rules, 6 J. Legal Stud. 65 (1977).

${ }^{4}$ John C. Goodman, An Economic Theory of the Evolution of the Common Law, 7 J. Legal Stud. 393 (1978).

${ }^{5}$ Robert Cooter, Lewis Kornhauser, \& David Lane, Liability Rules, Limited Information, and the Role of Precedent, 10 Bell J. Econ. 366 (1979). 
judges have insight beyond that displayed in their written opinions, and these opinions reflect a calculus of economic costs and benefits only in a narrow class of cases.

A broader class of cases would be susceptible to the second and third justifications. Furthermore, the second and third justifications parallel Adam Smith's invisible hand because "the wealth of nations" or efficiency is the unintended outcome of self-interested decisions by individuals. Deciding when to litigate and how much to invest in preparing your case may be motivated by self-interest, just as the decision to buy or sell a commodity. Litigants try to win their cases, not increase the law's efficiency, but the former may result in the latter. For example, if inefficient laws are litigated more frequently than efficient laws and if the probability that a precedent will survive a court test is independent of its efficiency, then the law may improve over time. This improvement would take place even if judges lack any insight into the efficiency of laws and even if litigants only follow their self-interest.

In this paper we assume that judges are ignorant in order to test the strength of the forces for legal evolution which operate without judicial help. We hope to make explicit a long tradition of informal discussion about evolutionary forces in the law by using the mathematics of biological evolution. The centerpiece of our theory is a matrix whose elements indicate the probability that a legal precedent will be revised; we model legal evolution by what is known as a Markov process. ${ }^{6}$

One of our conclusions is an impossibility theorem, which states that blind evolution will not cause the legal system to reach the best state or continually to improve itself. Eventually the process will settle down to a stable state in which each legal rule prevails a fixed portion of the time. Both "efficient" and "inefficient" or "best" and "worst" rules recur perpetually. The assumptions under which this pessimistic conclusion holds are general and appealing because, even at the expense of realism, they introduce a bias in the model in favor of evolutionary forces. The pessimism of our conclusion is mitigated somewhat by models which state that, while evolutionary forces cannot achieve a maximum on some standard of goodness (such as economic efficiency), they can improve the law relative to what it would be in their absence. For example, the stable state of the legal system becomes better as

\footnotetext{
${ }^{6}$ Markov processes are easily represented by physical or mechanical systems. Suppose there are three possible legal rules labeled red, yellow, and blue. The process of judicial decision is modeled as follows: each judge's chambers has three roulette wheels, one with a red ball, one with a yellow ball, and one with a blue ball. Each roulette wheel is tri-colored; the percentage of the area of the wheel which is red represents the probability that a judge will choose that rule on that wheel. When a case comes before a judge, he determines which rule currently prevails, say red. He then spins the wheel with the red ball and sees which color the ball lands on, say blue. He will announce the new rule as blue. In the next litigation he will spin the wheel with the blue ball which may be colored in different proportions than the wheel with the red ball.
} 
the frequency of litigation of bad laws increases. We shall prove these assertions by further theorems on Markov processes.

Part I presents the impossibility theorem, but without proof. Part II shows the artificial conditions under which evolutionary forces will lead to the most efficient state. Part III shows the limited way that litigation can improve the law without the help of judges. Part IV relates our model to others in the literature. Following some concluding remarks, the theorems are proved in a mathematical appendix.

\section{Impossibility Theorem on Evolution of Laws}

Our model is described by four assumptions which are rigorously formulated using mathematical notation in the appendix. First, we assume that there are a finite number of possible legal rules, which can be arranged in order from bad to good (assumption A1). It is convenient to index the set of possible legal rules $i=1,2 \ldots, n$, where a larger index number indicates a better rule; thus rule 1 is worst and rule $n$ is best. The standard of goodness may be precise and narrow, such as national income as measured in the national accounts, or it may be imprecise and broad, such as an index of the quality of life. Our conclusions are unaffected by whether the standard is a measure of economic efficiency or something else.

The evolutionary forces, however, must be consistent with the standard of goodness; that is, if efficiency is the standard and differential litigation the process, the frequency of litigation must depend on the "efficiency" of the rule. Similarly, a standard like the quality of life requires the frequency of litigation to depend on how the rule affects the quality of life. This point will become clearer as we develop the model.

At any point in time there is a rule in the finite set which describes the state of the legal system. Judges have an opportunity to change the state of the legal system by adopting a new precedent whenever litigation occurs. The next two assumptions describe how judges decide whether to change a rule which is litigated.

Our second assumption is that the probability of a judge abandoning one legal rule and adopting another depends upon the most recent legal decision, but not upon its predecessors (A2). ${ }^{7}$ In other words, the inclination of a judge to affirm or amend an existing precedent does not depend upon what hap-

\footnotetext{
${ }^{7}$ See note 6 supra. We have assumed that there are $n$ roulette wheels rather than three; this only makes the judge's chambers more crowded. Subsequent assumptions place some restrictions on the way the roulette wheels are colored.

We recognize that judges do not in fact use random processes to decide cases. We wish, however, to build as strong as possible a case for blind evolution in order to determine its impact under the most favorable conditions. As we show subsequently, even under these very favorable assumptions the law will not achieve and maintain efficient rules.
} 
pened before its adoption. In the actual court system past decisions eventually lose their influence over current decisions; A2 assumes that depreciation is instantaneous once a precedent is overturned. Let $p_{i j}$ be the probability that litigation of rule $i$ will cause the judge to replace it by rule $j$. In general $p_{i j}$ could be a variable whose value depends upon a long history of litigation; however A2 says that $p_{i j}$ is simply a number. This simplifying assumption makes legal evolution mathematically tractable, because it permits us to model legal evolution by a Markov process. ${ }^{8}$ There is a justification besides mathematical simplicity for making such an assumption. We want to restrict the learning ability of the judiciary in order to isolate independent forces for legal change. In effect A2 restricts judicial learning by limiting judicial memory.

Our third assumption is that there is a positive probability that a judge will replace any rule which is litigated by a neighbor on the scale of goodness (A3). For example, if the existing legal rule is fourth on the scale of goodness and it is litigated, then there is a positive probability that it will be replaced by rule 3 , which is worse by assumption $\mathrm{A} 1$, or by rule 5 , which is an improvement. We have already defined $p_{i j}$ as the probability that litigation of rule $i$ will lead to its replacement by rule $j$. Formally, A3 requires that $p_{i, i-1}>0$ and $p_{i, i+1}>0$ for $1<i<n$; it also requires that $p_{12}>0$ and $p_{n, n-1}$ $>0$. This assumption limits judicial insight into the goodness of the law by insuring that judges are willing to change both bad and good laws; furthermore, the change could be in either direction. Notice that the probability of an existing law being replaced by one which is better could be much higher than the probability of it being replaced by one which is worse (or vice versa) without violating A2, so long as neither probability is zero. Scope for judicial insight is limited but not absent. Notice also that A3 rules out a pure stare decisis doctrine, since we do not allow $p_{i i}$ to equal unity; however, $p_{i i}$ could be very close to unity. 9

\footnotetext{
8 We believe this assumption introduces a bias towards the conclusion that the common law through blind evolution tends to efficiency. Under a precedential system, one in which moreestablished rules are more likely to persist or ones in which "well-reasoned" opinions more strongly bolster rules than badly-reasoned ones, an inefficient rule might become entrenched and hence more likely to persist. Efficient rules, of course, might also become entrenched but in a system of "blind" justice there is no reason to believe that efficient rules will be more likely to be entrenched at the "outset" than inefficient ones.

For a mechanical description of Markov processes see note 6 supra. In the context of that example this assumption means that the judge uses the same set of roulette wheels in every litigation.

9 We can easily explain this assumption in the terms of our roulette-wheel example. Suppose that, instead of three colors, three rules, and three wheels, we have seven colors, rules, and wheels. Suppose further that the rules and the wheels are ordered in terms of the spectrum from red to violet. Assumption A3 says that every wheel has at least two colors in it. For example, the wheel with the green ball has the colors yellow, green, and blue on it because under A3 a green rule can only change to a rule "close" to it on the spectrum. All the wheels except the ones
} 
Assumptions 2 and 3 restrict the insight and learning ability of the judiciary in order to isolate the influence of differential litigation. We make these assumptions because we wish to characterize a process of blind evolution, not because we believe that they are true or that legal evolution is blind.

Our final assumption is that litigation is complete in the sense that there is a positive probability of litigation occurring at each point in time, regardless of the prevailing precedent (A4). This assumption insures that every precedent will be challenged; it is founded on the firm insight that someone stands to gain by a change in the legal rule, whether or not the rule is efficient. Let $q_{i}$ be the probability that rule $i$ will be litigated, assuming that $i$ is the prevailing rule. A4 is the assumption that $q_{i}$ is greater than zero for all $i{ }^{10}$

The probability that the existing rule will change depends upon both the probability that it will be litigated, and the probability that a judge will change it when litigated. The overall probability can be obtained by combin-

with the red and violet balls have at least three colors on them. The wheel with the red ball has at least red and orange because there is no "infrared" rule, while the wheel with the violet balls has at least indigo and violet on it because there is no "ultraviolet" rule. A doctrine of pure stare decisis would mean that each wheel would be colored completely in the same color as the ball; for example, the wheel with a green ball would be completely green.

Assumption A3 is unnecessarily restrictive. Mathematically, we require only indecomposability and aperiodicity of the transition matrix. Indecomposability means that for every pair of states $i$ and $j$, there is a positive probability that the law will change from state $i$ to state $j$ in some finite amount of time. In a periodic Markov process the set of states can be divided, starting from any set $B_{1}$ into disjoint subsets $B_{1}, \ldots ; B_{d}$ such that the process never returns to set $B_{1}$ in less than $d$ steps and always returns on the $d$ th step. A simple random walk on the real line is a periodic process: Suppose the probability of moving from one integer to the next greatest integer is $1 / 2$ and to the next smallest integer $1 / 2$. Then the motion is periodic with the even integers and odd integers, the two disjoint subsets of states. An aperiodic motion is one that is not periodic. Assumption A3 coupled with an assumption that a diagonal element is nonzero is sufficient (but not necessary) to assure that the Markov process under consideration is indecomposable and aperiodic.

It is worth noting that our model holds even if there are some possible inefficient states that would never be adopted by a court. Our model requires only that there be some set of rules, efficient and inefficient, considered feasible (or viable) by the legal system. Only this feasible (or viable) set of rules need conform to our assumptions.

$1^{10}$ Our system of roulette wheels described what happened once the case got into court. Assumption A4 describes when a case is litigated. One can imagine each pair of potential litigants deciding to settle or to litigate by throwing a dart at a dart board. Again we would require as many dart boards as rules. Each dart board would have two colors on it: black (litigate) and white (settle). The board with the red dart need not have the same percentage of its area white as the board with the blue dart. Assumption A4 says that every board has some black on it.

As noted in the next paragraph of the text this system of dart boards and roulette wheels can be consolidated into a system of roulette wheels like that described in notes 6-9 supra. The effect of the dart boards is to increase the likelihood that a rule will not change because if a rule is not litigated it surely will not be changed. Thus the augmented system of wheels looks very much like the unaugmented system described in notes 6-9; more of each wheel has the color of the ball used on it. 
ing the $q$ 's and the $p$ 's as shown in the appendix. The result is a transition matrix $^{11}$ for the legal system, which describes the probability that any existing rule $i$ will change to rule $j$. This matrix takes into account both the probability of litigation occurring and its likely outcome.

A characteristic of the processes which we examine is that their long-term behavior is independent of the legal rule from which they begin; the starting point eventually loses all influence. We use the term "stable state" to describe the situation towards which the Markov process is tending. In the stable state each rule of the legal system prevails a fixed percentage of the time. The proportion of time that a rule prevails may also be described as the probability that it is prevailing. If the probability is 1 for rule $i$, then it prevails 100 percent of the time. In that case all of the other rules are the precedent for a finite length of time, whereas one rule persists as the precedent for an infinite amount of time. The stable state then consists of rule $i$ prevailing for all but a finite amount of time. Alternatively, there may be several rules for which the probability is neither 1 or 0 , in which case each of these rules will be the precedent an infinite number of times. Another way to say this is that the time expected to elapse before one of these rules recurs as the precedent is finite.

Now we can state our impossibility theorem:

THEOREM 1. Assuming A1-A4, the legal system tends towards a stable state in which each rule prevails a positive proportion of the time.

This theorem states that every legal rule-from best to worst-is expected to become the precedent, be replaced, and become the precedent again. The time expected to elapse between recurrences is finite. The legal system never settles down to a situation in which the best legal rule prevails forever and the inferior rules never recur; the worst legal rule is expected to recur. This theorem is true even if bad rules are litigated much more often than good rules, and even if judges are more likely to replace bad rules by good rules than to do the opposite. In brief, the course of evolution does not lead to an equilibrium that is best or nearly best. ${ }^{12}$

11 Every finite Markov process can be described by a matrix of probabilities. The matrix is called a transition matrix as it describes how the process (in our case the process of legal change) evolves from period to period. The probability number recorded in the $i$ th row and $j$ th column of the matrix represents the probability that the process starting in state $i$ will move in the next time period to state $j$.

12 Theorem 1 may be expressed in the terms of our roulette-wheel example. A stable state is simply another roulette wheel (say, with a white ball) with which we may replace the complex system of roulette wheels used by the judge (in note 6 , the three-wheeled system). Our theorem says that the legal systems described can be replaced by one roulette wheel and that this roulette wheel will have all (three) colors on it; hence in every litigation there is some chance of getting each of the possible legal rules. 


\section{Possible But Implausible Evolution}

Suppose that no one ever litigates a rule that is best. In that case we must replace our assumption of complete litigation (A4) by an assumption of partial litigation: Assume that every rule except the best is litigated with positive probability and the best is never litigated $\left(\mathrm{A4}^{\prime}\right)$. The consequence of this assumption is that the best legal rule, if it is ever selected by a judge, is never overturned. In the course of time we expect the legal system to reach the best state and never leave it; conversely we expect the states which are not best to cease recurring as the precedent. We prove the following theorem in the Appendix:

Theorem 2. Assume A1-A3 and A4'; then the legal system tends towards a stable state in which the best rule prevails 100 percent of the time.

This theorem states that we expect the inferior rules to eventually cease recurring, whereas the best rule persists forever. The result should not be surprising given the assumption $\mathrm{A} 4^{\prime}$. After all $\mathrm{A} 4^{\prime}$ says that once the legal system finds the best rule it will recognize its accomplishment by never changing the rule. Since every other rule is likely to be changed, and since some of these rules could be changed to the best rule, we would expect that in some finite, though possibly greatly extended time, the best rule would be found by the courts. ${ }^{13}$

We consider the assumption of partial litigation $\left(A 4^{\prime}\right)$ to be implausible because someone always stands to gain by revising the law, but other theorists disagree. For example, Rubin has constructed a model in which the inefficient rule is litigated and the efficient rule is never litigated. ${ }^{14} \mathrm{We}$ shall compare our model with Rubin's in detail and defend our own viewpoint in Part IV.

\section{How Litigation Can Improve the LaW without the Help of Judges: Comparative Statics of Markov Processes}

We have proved that evolutionary forces are insufficient to cause the legal system to adopt and retain the best legal rule without the help of judges. In this part we relieve somewhat the pessimism of this conclusion. At least, for simple processes of legal change, evolutionary forces can improve the legal system relative to what it would be otherwise.

Our first task is to define "improvement." In A1 we assumed that legal rules can be set in order from worst to best, and we indexed these rules

\footnotetext{
${ }^{13}$ Assumption A4' says that the dart board associated with the best rule (say, the violet rule) is all white; once a ball of some color lands on violet the litigants never resort to the set of wheels in the judge's chambers.

${ }^{14}$ Rubin, supra note 3.
} 
$1,2, \ldots, n$, in order of increasing goodness. A stable state is described by a probability distribution over these rules, so our task is to define conditions under which one probability distribution is better than another. One probability distribution is unambiguously better than another if better rules are more likely to occur and worse rules are less likely to occur. ${ }^{15}$ We make this idea precise with the help of some additional notation. Let $x=$ $\left(x_{1}, x_{2}, \ldots, x_{n}\right)$ and $y=\left(y_{1}, y_{2}, \ldots, y_{n}\right)$ be two different vectors, assigning probability to legal rules $1,2, \ldots, n$; for example, the probability that the legal system has rule 2 is $x_{2}$ when $x$ is the probability distribution. We shall say that $x$ is better than $y$, denoted $x R y$, if $x$ assigns more probability to good rules and $y$ assigns more to bad rules. More precisely, $x$ is better than $y$ if we can obtain distribution $x$ from $y$ by shifting some of the probability density in $y$ to the right, thus assigning more weight to better rules. The relation $R$ is fairly intuitive. Think of the vectors $x$ and $y$ as two different gambles, each with the same possible outcomes but different probabilities. When we shift some probability density to the right, we make a better payoff more likely. Anyone who agreed with the ordering of rules $1,2, \ldots, n$ in assumption $A 1$ would prefer gamble $x$ over gamble $y .{ }^{16}$

The legal processes which we model are all characterized by a unique stable state-that is a probability vector $x=\left(x_{1}, \ldots, x_{n}\right)$ each component $x_{i}$ of which indicates the percentage of time the legal system spends in state $i .{ }^{17}$ If $A$ and $B$ are transition matrices with stable states $y$ and $x$ respectively, then we say that the legal system represented by $B$ is better than the legal system represented by $A$ if and only if $x R y$. To compare legal systems, then, one need only compare the stationary distributions to see whether one can be obtained from the other by shifting probability to the right. ${ }^{18}$

15 Suppose in the three-wheel example, blue is better than yellow which is better than red. Suppose further we had two jurisdictions 1 and 2 with two distinct systems of roulette wheels (that is the percentage of the area of each wheel with a particular color differs in the two jurisdictions) but each system of wheels satisfied Al through A4. The process in jurisdiction 1 could then be represented by wheel $W_{1}$ and the process in jurisdiction 2 could be represented by wheel $W_{2}$. Jurisdiction 1 would be better than jurisdiction 2 (or it would be an improvement to substitute jurisdiction 1's system for jurisdiction 2's) if the percentage of red area in $W_{1}\left(R_{1}\right)$ were less than the percentage of red area in $W_{2}\left(R_{2}\right)$ and $R_{1}$ plus the percentage of yellow area in $W_{1}$ $\left(Y_{1}\right)$ were less than or equal to $R_{2}$ plus the percentage of yellow area in $W_{2}\left(Y_{2}\right)$.

16 Our definition of " $R$ " is ordinalist in the sense that it assumes legal rules can be ordered by goodness, but nothing is known about how much better one rule is than another. If $u$ is monotonically increasing in rules $i=1,2, \ldots, m$, then $[x R y] \Rightarrow[x \cdot u(i) \geq y \cdot u(i)]$ for every $u$. The inequality is preserved under monotonic transformations of $u$. An alternative approach would assume that probability distributions over legal rules can be ordered cardinally; in that case we would say that the legal system has improved if its expected utility is higher. The expected utility approach has the advantage of ordering every possible probability distribution, whereas our approach cannot order some pairs; the disadvantage of the expected utility is that it requires much more information.

${ }_{17}$ The systems considered in this paper all satisfy the conditions of Theorem 6.27(a) in Leo Breiman, Probability and Stochastic Processes 180 (1969). That theorem establishes existence and uniqueness.

18 See notes 12 and 15 supra. 
Our aim in this part is to discover conditions under which evolutionary forces will improve the legal system. The conditions under which this is true are best summarized by a theorem on transition matrices. The transition matrix combines the probability that a state will be litigated with the probable decision of the judge. For example, let $A$ be a transition matrix, each row of which has the form $a_{i}=\left(a_{i 1}, a_{i 2}, \ldots, a_{i n}\right)$. An element $a_{i j}$ in row $i$ indicates the probability that rule $i$ will be overturned at any point in time when it is the precedent and replaced by rule $j$.

We shall require that the transition matrix satisfy a special characteristic, namely each row must be better than the preceding row: formally, the matrix $B$ with rows $b_{i}$ must satisfy $b_{n} R b_{n-1} R \ldots R b_{2} R b_{1}$. For such a matrix the likelihood that the legal rule will be good in the next period is higher if the precedent is good than if it is bad; in other words the expected value of the gamble represented by the legal process is higher if the existing precedent is better. Some features of real legal systems might produce this result. ${ }^{19}$

We have seen that each row of the transition matrix is a probability vector. Suppose that we shift some of the probability density in row $k$.of matrix $A$ to the right, thus creating a new matrix $B$ such that $b_{k} R a_{k}$. We are contemplating two legal processes $A$ and $B$ which are identical except for the fact that when the legal system is in state $k$ the gamble presented by process $B$ is better than the gamble presented by process $A$; legal rule $k$ is more likely to be replaced by a good rule in process $B$ than in $A$. The shift from $A$ to $B$ could have several different causes. For example, judges may have gained more insight into how to improve rule $k$, or judges may be more inclined to overturn rule $k$ because litigants favoring a better rule invest more in litigation. Alternatively, the shift may result from an increase in the rate of litigation of $k$ where $k$ is a bad rule. Whatever the cause, we can prove that the stable state is better for $B$ than $A$ provided that the condition which we described in the preceding paragraph is satisfied:

Theorem 3. Assume A1-A4 and also assume that the legal process is one in which the better the precedent the more likely that the legal rule will be good in the next period. If a change occurs so that some legal rule is more likely to be replaced by a good legal rule than formerly, then the changed legal process tends towards a stable state which is better than the stable state of the original process. Formally, if $A$ and $B$ are Markov matrices satisfying (i)

\footnotetext{
19 The condition of the text is nonintuitive; we shall not attempt to adapt our system of roulette wheels to it. It is not hard to see why real legal processes might satisfy this condition. If the judiciary is reluctant to make large changes abruptly, then it will be impossible to jump from a very bad state to a very good state, in which case similar laws will be likely to succeed each other as required. In effect our condition requires that legal rules change incrementally or by small adjustments; judicial inertia lead to a legal process with the desired characteristic. Of course judicial perceptions of when rules are close together need not coincide with the "goodness" ordering we have imposed on our rules. See note 20 infra for further discussion.
} 
$b_{i} R a_{i}$ for each $i$ and (ii) $b_{n} R b_{n-1} R \ldots R b_{2} R b_{1}$, then $x R y$, where $x$ is the stable distribution of $B$ and $y$ is the stable distribution of $A$.

As an example of a pair of matrices which satisfy conditions (i) and (ii), consider the following:

$$
A=\left[\begin{array}{lll}
.5 & .5 & 0 \\
.2 & .6 & .2 \\
0 & .3 & .7
\end{array}\right] \quad B=\left[\begin{array}{lll}
.5 & .5 & 0 \\
.2 & .6 & .2 \\
0 & .2 & .8
\end{array}\right]
$$

By shifting density to the right we can obtain row 3 of matrix $B$ from row 3 of matrix $A$; rows 1 and 2 are identical in $A$ and $B$; so condition (i) of Theorem 3 is satisfied. By shifting density to the right we can obtain row 2 from row 1 of matrix $B$, and row 3 from row 2 of $B$, so condition (ii) in the theorem is satisfied. It follows from Theorem 3 that the stable state of $B$ is better than the stable state of $A$; in fact numerical calculation shows that the stable state of $A$ is $y=(.19 .49 .32)$ and the stable state of $B$ is $x=(.16 .42$ .42). That is, in $A$ the legal process represented by rule 1 will prevail 19 percent of the time, rule 2 prevails 49 percent of the time, and rule 3 prevails 32 percent of the time; while in the process represented by $B$, rule 1 prevails 16 percent of the time, rule 2 prevails 42 percent of the time, and rule 3 prevails 41 percent of the time. Note that in neither system does rule 3 prevail even half the time.

We can prove a much stronger theorem for matrices which have symmetry properties such as those in the preceding example. Let us specify some additional assumptions on the behavior of judges and litigants which lead to this stronger theorem. Specifically, assume that courts will only change a law to one next to it in the order of goodness; ${ }^{20}$ for example, judges may change rule 5 into 4 or 6 , but they will not change 5 into 3 or 7 . We insure that judges lack insight into how the law ought to be changed by assuming that the probability of a judge making the law better is the same as the probability of making it worse. Finally, we keep the problem mathematically simple by assuming that judges are more inclined to revise a bad law than a good law, which would occur if litigants invest more in building a case against bad laws. Formally, these conditions can be written for all $i$ and $j$ (A5):

$$
p_{i j}=0 \text { if }|i-j|>1 \quad \text { (inertia); }
$$

20 There is a slight inconsistency in our denotation of justice as blind and our assumption that the law moves in small steps. The notion of closeness we use is one consistent with the ranking of goodness. The legal system apparently recognizes the "goodness" ranking in making changes but does not recognize it as a criterion for choosing a direction in which to move. Technically, the only assumption we need is that for any two states of the law $i$ and $j$, state $j$ can be reached from state $i$ in some finite number of steps with positive probability. Thus, a more felicitous formulation of the small-step idea would be to postulate a ranking actually used by the judges or some commonsense notion of closeness in the formulation of the rules and require that ranking to meet the technical condition (called indecomposability) noted above. 


$$
\begin{array}{cl}
p_{i, i+1}=p_{i, i}-1 & \text { (no insight; symmetry); } \\
p_{i i}<p_{j j} \text { if } i<j & \text { (stability of good laws). }
\end{array}
$$

The transition probabilities are a consequence of both the decisions of judges and the decision to litigate; A5 specifies what judges do, but we still have to specify the behavior of litigants. Suppose that we retain the idea that litigation is complete, but we make the specific assumption that bad laws are litigated more often than good laws (A6); more precisely we assume for all $i$ and $j$

$$
\begin{aligned}
& 1>q_{i}>0 \text { and } \\
& q_{i}>q_{j} \text { if } i<j .
\end{aligned}
$$

Conditions A1-A6 define a specific model whose properties are relevant to recent arguments about legal evolution. We can prove the following theorem:

Theorem 4. Assume A1-A6. The legal system has a stable state such that the proportion of time during which each rule prevails increases monotonically with the goodness of the rule. Furthermore, an increase in the probability of litigating any rule $j$, where $j<n$, affects the stable state in such a way that (i) the proportion of time increases during which any rule prevails other than rule $j$, and (ii) the better the rule, the larger is the increase in the proportion of time it prevails.

The first part of this theorem states that the legal system tends towards a condition in which it is more often good than bad. The second part of the theorem states that any increase in the rate of litigation of bad rules will improve the legal system. For example, if it becomes more likely that rule 1 -the worst rule in the legal system-will be litigated, then the theorem tells us that it becomes more likely that the legal rule prevailing at any point in time will be some rule other than 1 ; furthermore, the greatest increase in probability resulting from this change is the increased likelihood of the best rule prevailing.

Priest stresses the idea that differential litigation causes the legal system to be more efficient than judges want. ${ }^{21} \mathrm{We}$ can interpret this proposition in terms of Theorem 4 . Let $\left[p_{i j}\right]$ be the matrix describing how judges change the law whenever cases arise. This matrix has a stable state which characterizes the legal system when every rule is litigated at the same rate. The transition matrix for the actual legal system is a result of the interaction between judicial wants and differential litigation. If differential litigation and judges' desires satisfy the conditions given in Theorem 4 , then the stable state of the actual legal system lies to the right of the stable state of the matrix $\left[p_{i j}\right]$. In

\footnotetext{
${ }^{21}$ Priest, supra note 3.
} 
this sense the actual legal system is better or more efficient than judges want it to be.

Goodman has argued that the legal system will contain more efficient laws than inefficient laws at any point in time, because it is more likely that an inefficient law will be overturned as a consequence of litigation. ${ }^{22}$, The idea that an inefficient law is more likely to be overturned is captured by the third part of assumption A5 $\left(\phi_{i i}<p_{j j}\right.$ if $\left.i<j\right)$. This assumption, together with the others named in Theorem 4, leads to the conclusion that the legal system tends towards a stable state in which the proportion of time that a legal rule prevails is an increasing function of the rule's efficiency. Goodman's argument is similar to this interpretation of Theorem 4; in the next part there is a more detailed comparison of our results with other theories in the literature.

Our Theorem 3 states conditions that more complex legal systems must meet if the assertions of Priest and Goodman are to hold. Unfortunately condition (ii) of Theorem 3 is a technical one for which we have very little intuition. It is therefore difficult to say whether actual legal processes, which are surely more complex than those discussed in Theorems 3 and 4, conform to the conditions of Theorem 3. Evaluation of Priest's and Goodman's assertions must await some means of "verifying" or interpreting condition (ii).

\section{Does Litigation Vary with the Efficiency of a Rule of Law?}

We have shown how to apply mathematical models of biological evolution to the law. Our theorems suggest that, if legal processes are simple, evolutionary forces can improve the law relative to what it would otherwise be. In particular, we have shown what consequences to expect if inefficient laws are litigated more frequently and if more is invested in overturning them than in defending them. However, we have offered no argument to prove that the frequency or intensity of investment in litigation varies systematically with the efficiency of the law. We have shown the consequences of evolutionary forces without demonstrating their presence in the law. In this part we discuss theories of litigation which bear upon the question of whether legal evolution is at work.

It is simplest to model litigation as a two-person bargaining game. ${ }^{23}$ For example, Table 1 shows the payoff matrix of a typical liability case where $x$ has injured $y$. We assume that the damages suffered by $y$ are 10 . If $x$ pays the damages and settles out of court, then $x$ pays 10 and $y$ receives 10 , so the joint costs are nil; similarly the joint costs are nil if $y$ does not litigate and $x$ does not pay compensation. However, if litigation occurs then each party

22 Goodman, supra note 4.

23 Technically the games in question are two-person, nonzero sum games with communication and side payments. 


\begin{tabular}{|c|c|c|c|}
\hline & & \multicolumn{2}{|c|}{$X$ (injurer) } \\
\hline & & $\begin{array}{c}\text { Pay } \\
\text { Compensation } \\
-15\end{array}$ & $\begin{array}{c}\text { No } \\
\text { Compensation } \\
-5\end{array}$ \\
\hline & Litigate & 5 & -5 \\
\hline \multirow[t]{2}{*}{$Y$ (victim) } & & -10 & 0 \\
\hline & Settle & +10 & 0 \\
\hline
\end{tabular}

TABLE 2

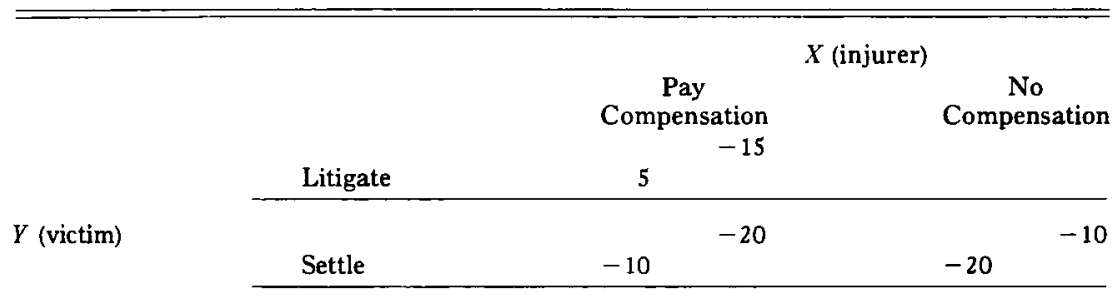

spends 5 on litigation. The joint costs of litigation are -10 , whatever the outcome of the litigation, as can be seen by adding the two numbers in each cell of the first row. The surplus from cooperating and settling out of court is 10 , which is the difference between the joint costs of litigating and settling out of court. The surplus is what is lost or the efficiency cost of not cooperating; alternatively, the surplus is what the players gain as a reward for cooperating.

According to one tradition in game theory, players will always minimize their joint costs. ${ }^{24}$ This assumption is called "group rationality" because the players minimize costs falling upon the whole group of bargainers. Group rationality would never result in litigating cases, like those represented by Table 1 , where litigation is costly.

In some circumstances the judge may impose a settlement more cheaply than the litigants can achieve through negotiation. In such cases we say that the cost of negotiating a settlement exceeds the cost of litigation, or settlement costs exceed litigation costs. We have modified Table 1 to produce this result. In the new situation depicted by Table 2 , the cost of settling without compensation is -20 . Furthermore, the cost of negotiating a settlement with compensation is -10 to each party, whereas litigation can achieve the same result at a cost of -5 to each party. Under these circumstances group

\footnotetext{
${ }^{24}$ See R. Duncan Luce \& Howard Raiffa, Games and Decisions, ch. 6 (1957). It is not clear whether this tradition was ever intended to serve as the basis of a predictive theory of bargaining; rather it may have been intended to show what outcomes are fair or reasonable.
} 
rationality would require litigation, providing that the likelihood of a judge ruling for the plaintiff is high, which we indicate by leaving the northeast cell blank. In brief, litigation occurs because the surplus from cooperation is negative, due to the fact that settlement costs exceed litigation costs.

When the activities that result in the accident are recurrent or ongoing, two types of settlement are possible. Parties may divide in some way the loss that occasioned the immediate dispute; or they may, in addition, agree that precautions will be taken. Generally, an agreement of the latter variety will provide for precautions not mandated by the law; for instance, the agreement may require precautions of the party who is not liable under existing law. The parties may thus be said to "negotiate around the law."25 An important aspect of any theory of litigation is whether it permits negotiating around the law.

Rubin adopted this theory of litigation (with a "no negotiating around the law" assumption) in the first published article with a formal analysis of legal evolution. ${ }^{26} \mathrm{His}$ basic model of litigation is a two-person bargaining game in which the players minimize joint costs. The joint costs are the costs of accidents, which include both damages and expenditures to prevent them, and transaction costs, which consist of court costs and settlement costs. Rubin assumes that the legal system has two possible rules regarding accidents, one of which assigns liability to the first person and the other assigns it to the second person. The rule of law is efficient when it assigns liability to the person who can prevent accidents at least cost. Rubin assumes that the cost of negotiating around the law is prohibitive, but the cost of negotiating a settlement consistent with the law is less than court costs. ${ }^{27}$ If

${ }^{25}$ We have glossed over a significant difference between settlement and litigation. In the game represented by Table 1, the parties save 10 cost units by settling. Any settlement would save the identical amount. Further the victim would be willing to accept any settlement amount greater than 5 , the amount he receives in a successful litigation, while the injurer will be willing to pay any amount less than 15 , the amount he pays in an unsuccessful litigation. The actual settlement (or payment to the victim) can be any number between 5 and 15 .

In settlements in which the parties have negotiated around the law, the party taking extra precautions will be incurring a cost but may not be receiving the benefits. For example, in a defective-product situation an agreement under which manufacturers took more care than required by law would impose extra costs on them but, absent a transfer of incomes from consumers, the manufacturers would receive no benefit. If taking the extra care is efficient, the consumers, of course, would be willing to pay an appropriate amount. While a settlement permits such payments, litigation does not. When the rule of law is changed, there may be a net social gain but one party may suffer a loss. The party which loses will have an incentive to litigate to change the law away from efficiency.

${ }^{26}$ Rubin, supra note 3.

${ }^{27}$ Rubin does not justify his assumption that negotiating around the law is prohibitively expensive. We think it likely that, in those situations where negotiating around the law is impossible, either one party will not have a long-term interest in the subject of litigation or private and social interests will not be coincident. Consider, for example, accidents caused by defective products. To conform to Rubin's model, consumers in general rather than a particular 
the precedent is efficient, then there is no reason to change the law or negotiate around it, so the parties settle in order to save court costs. If the precedent is inefficient, then accident costs can be reduced by successful litigation; negotiating around the law is prohibitively expensive by assumption. The choice is between a settlement which retains the inefficiency or litigation to change the law. Litigation occurs if court costs are less than the cost of the inefficiency; otherwise there is a settlement. ${ }^{28}$ In summary, efficient laws are litigated if court costs are relatively low ${ }^{29}$

In our opinion the connection between frequency of litigation and efficiency of the precedent must be established econometrically. Rubin's a priori model suggests propositions to test but provides no answers warranting belief. Furthermore, it is our judgment that the assumption of group rationality used by Rubin and others will not provide an adequate foundation for an empirical theory of litigation. Much litigation does not arise from group rationality, despite its mathematical elegance. Litigation is often caused by bargaining breakdowns, vengefulness, or strategic behavior,

consumer must be regarded as the party adverse to the manufacturer. In a specific litigation, consumers are represented by two people; the injured consumer represents the interest in compensation for the immediate accident and the plaintiff's attorney represents consumers' long-term interest in the rule of law. As neither the attorney nor the plaintiff can bind other consumers to an agreement negotiated around the law, such agreements probably are prohibitively expensive. The interests of the plaintiff's bar, however, are not necessarily coincident with those of consumers or with society. This deviation of private and social interests would prevent the law, in Rubin's model, from achieving efficiency.

${ }^{28}$ We can translate the verbal argument of this paragraph into Rubin's mathematical notation. If player $A$ takes precautions and $B$ takes none, then the joint future costs of accidents and accident avoidance are designated $T_{A}$. If the existing rule of law assigns liability to $A$ and there is a settlement of past costs, then joint costs will continue to be $T_{A}$. If the current case is litigated, then the joint court costs will be $2 C$. There is a probability $R$ that the current precedent will be reaffirmed, in which case the future costs will remain $T_{A}$; there is a probability $(1-R)$ that the precedent will be overturned and liability assigned to $B$, in which case he will take precautions, resulting in joint future costs denoted $T_{B}$. So the expected joint costs from litigation are $\mathrm{RT}_{A}+(1-R) T_{B}+2 C$. The players litigate whenever the expected joint costs are less than the joint costs of settlement:

$$
R T_{A}+(1-R) T_{B}+2 C<T_{A}
$$

or equivalently

$$
(1-R)\left(T_{A}-T_{B}\right)>2 C .
$$

If court costs $C$ are positive and the current rule of law is efficient $\left(T_{A}<T_{B}\right)$, then this inequality cannot be satisfied. If $R$ is positive and $C$ is small, and if the current rule of law is inefficient $\left(T_{A}>T_{B}\right)$, then the inequality will be satisfied.

${ }^{29}$ Rubin's assumption is a special case of our assumption of partial litigation (A4'). A4' includes Rubin's assumption that efficient rules are never litigated but $\mathrm{A} 4^{\prime}$ is more general because it allows for inefficient rules to be settled. A4' is less general than the assumptions implicit in Rubin's model because he allows positive costs of litigation. In our discussion the cost of litigation implicitly has been assumed to be zero. This introduces in our model another bias towards efficiency. 
which increase joint costs. ${ }^{30}$ These considerations are likely to cause litigation whether the precedent is efficient or inefficient.

There is another a priori argument which has been used to conclude that the law will evolve towards efficiency. Posner has described a simple mechanism by which litigation is more likely when the stakes in the case are large. Litigants do not know in advance which way a case will go; as a consequence the effect of litigation on costs is an expected value, rather than a certain value. This value may be described as the product of the probability that the law is changed times the actual joint benefits (stakes) from changing it. If the subjective probability that the courts will change the law is independent of the size of the stakes, then the expected benefits will be greater when the stakes are large. Litigation cannot occur unless the expected benefits from it exceed the actual court costs. It follows that, for a given subjective probability level, the set of circumstances which lead to litigation is larger when the stakes are high than when they are low. ${ }^{31}$

Priest first saw the implications of Posner's argument for efficiency in the law. The stakes or joint benefits of changing the law are higher when the law is inefficient, so it follows that inefficient laws will be litigated more fre-

30 This point is discussed at length in Robert Cooter, The Cost of Believing Coase's Theorem (1978) (unpublished mimeographed paper).

${ }^{31}$ In Posner's model litigation arises when the surplus from cooperation is negative, but the source of the negative surplus is differing expectations about the outcome of litigation, rather than transaction costs. These cryptic remarks do not capture the flavor of Posner's theory, but a systematic exposition would take us far from our main point. Posner's actual argument is more complicated than suggested by this brief statement. If we think of the plaintiff as offering to sell release from the suit, then he will not sell for a price which is less than his expected gain from the suit, and the defendant will not pay more than the expected cost of the suit to him. In this framework a sufficient condition for litigation is that the maximum amount the defendant would pay to be released from the suit is less then what the plaintiff expects to gain from it. This is equivalent to saying that the expected surplus from cooperation is negative. See Richard $A$. Posner, An Economic Approach to Legal Procedure and Judicial Administration, 2 J. Legal Stud. 399 (1974), especially at 419 n. 29 .

In a recent paper Landes and Posner present a model of the litigation/settlement choice which includes a more sophisticated notion of precedent. William $M$. Landes \& Richard A. Posner, Adjudication as a Private Good, $8 \mathrm{~J}$. Legal Stud. 235 (1979). Their interesting model implies that efficient rules will be litigated more often than inefficient rules. In note 33 infra we offer a model with a less-sophisticated concept of precedent that leads to the same result. We do not examine Landes and Posner's model at great length here because, in this part, we are more interested in the bargaining assumptions that underlie models of the litigation/settlement choice than in explicating the concept of precedent.

Landes and Posner employ a joint-cost-minimizing assumption. (See their equation (11), id. at 269.) The relationship of size of the surplus to the "likelihood" of litigation, however, is more complex than in Rubin's world. From Landes and Posner's equations (14) and (15) (id. at 271) we may conclude that (1) litigation will never occur unless $\delta>1-p /(1-p)$ (where $\delta$ is a measure of the "precedential significance" of winning relative to losing-see $i d$. at $271 \mathrm{n} .82-$ and $p$ is the probability that liability will be imposed efficiently) and (2) if $\delta>-p /(1-p)$, the larger the surplus, the wider is the domain of litigation costs under which litigation will occur. That is, as the surplus increases, the greater is the incentive to improve the rule (as long as people, through observation of the precedent, believe improvement is possible). 
quently than efficient laws. Priest's theory makes this fact the mechanism for evolution in the law. ${ }^{32}$

Posner's argument is interesting and worthy of investigation but a model based upon an equally simple mechanism leads to the opposite conclusion. In most cases precedent will be sustained, not overturned. Under an inefficient rule of law, litigation will result most likely in court-enforced inefficiency. This fact provides an incentive to settle out of court. However, if the precedent is efficient, the parties have less to fear from a court settlement; so litigation is more probable. Given a choice between private mediation and mediation by the courts, the parties are more apt to choose the courts if the precedent which applies to their case is efficient. This argument suggests that more efficient laws will be litigated more frequently. ${ }^{33}$ In the

32 Priest stresses that differential rates of litigation make the law more efficient than it would be otherwise, and in this sense the efficiency of the law is independent of the will of judges. See Priest, supra note 3.

${ }^{33}$ We can formalize this argument so that it retains most features of the theories which we wish to criticize but leads to the opposite conclusion. In particular we retain the assumption that plaintiff and defendant are minimizing their joint costs. In the simplest model we need not, as Posner and Rubin do not, raise the issue of how these costs are distributed, so it may be helpful to think of the plaintiff acting as a broker by deciding whether or not to litigate. Let $T_{e}$ be the joint cost of accidents and accident avoidance by the two parties when the rule of law is efficient, and let $T_{n}$ indicate these costs when the rule of law is not efficient; hence $T_{n}>T_{e}$. Let $\tilde{p}$ be a random variable indicating the plaintiff's subjective probability that the existing precedent will be sustained. (That is, the plaintiff's beliefs are assumed to be distributed in some fashion over the interval $(1 / 2,1)$. The belief that the existing precedent will be sustained is determined by drawing from this distribution.) Finally, let $C$ be court costs. If the existing precedent is not efficient, then the plaintiff expects joint costs from litigation to be $\tilde{p} T_{n}$ $+(1-\tilde{p}) T_{e}+C$, whereas the expected costs when the law is efficient are $\bar{p} T_{e}+(1-\tilde{p}) T_{n}+C$. Settlement is the alternative to litigation. Assume that the cost of reaching a settlement corresponding to the efficient rule of law is less than the cost of settlement corresponding to the inefficient rule of law; in other words, the settlement which minimizes joint costs results in costs $T_{e}+S$, where $S$ indicates the cost of negotiating an efficient settlement. Litigation occurs when its expected costs are less than the cost of the best settlement:

$$
\begin{aligned}
& \text { Inefficient precedent: } \bar{p} T_{n}+(1-\bar{p}) T_{e}<T_{e}+S-C \\
& \text { Efficient precedent: } \bar{p} T_{e}+(1-\bar{p}) T_{n}<T_{e} S-C .
\end{aligned}
$$

By definition $T_{n}>T_{e}$, and by assumption it is more likely that the precedent will be sustained than overturned, $p>(1-p)$; it follows that the second inequality will be satisfied over a wider range of subjective beliefs for given costs $T_{n}$ and $T_{e}$. Similarly for fixed subjective probability beliefs, the second inequality will be satisfied over a wider range of ratios of $T_{n} / T_{e}$ than the first. The set of circumstances in which efficient rules will be litigated is therefore larger than the set in which inefficient rules will be litigated.

This simple theory formalizes the idea that litigants are more likely to appeal to the law to resolve their disputes when they believe that courts will apply an efficient rule to their case, and they are more likely to resolve their disagreement privately when they believe that the courts will enforce an inefficient solution. If we make the model more realistic, then we will discover forces which work in the opposite direction. For example, in a general model the cost of an efficient settlement $S$ should be a function of the precedent; the probability distribution $\bar{p}$ may differ according to the law's efficiency; finally, the defendant may have different subjective probabilities $\tilde{p}$ than the plaintiff. At a deeper level, the whole approach of basing the decision to litigate on joint-cost minimization may be wrong. We are not claiming that our simple model is 
absence of empirical evidence there is not much basis to choose between this argument and Posner's. The connection between the size of the surplus and the likelihood of litigation is an open empirical question which cannot be answered by a priori models.

We have not found a compelling reason to think that inefficient laws will be litigated more frequently; a separate argument advanced by Goodman is that more will be spent in preparing the case against inefficient laws than in defending them. ${ }^{34}$ Goodman argues that (1) litigants seeking to overturn inefficient laws have more to gain by winning than would be lost by those who benefit from retaining them; and (2) if litigation is viewed as an investment, then litigators will be willing to spend up to the point where the expected benefits equal the costs; (3) consequently, they will be willing to spend more on overturning inefficient laws than defending them; and (4) presumably judges are influenced by the quality of the case that each side makes, so the courts will tend to overturn inefficient precedents. In the preceding part we noted that this argument is captured by the third part of assumption A5 $\left(p_{i i}<p_{j j}\right.$ if $\left.i<j\right)$, which leads to the conclusion in Theorem 4 that better laws will prevail a higher percentage of the time.

Notice that Goodman's argument does not assume joint-cost minimization, which is why it is more appealing to us than the models of Priest and Rubin. However, it remains to be determined whether the force towards efficiency identified by Rubin is swamped by forces working in the opposite direction. We suspect that the parties who have invested most heavily in litigation have been large institutions which internalize more of the benefits to be derived from favorable court actions, as opposed to individuals who only realize a small part of the benefits from their investment. In order to determine whether more is spent on overturning inefficient laws than in defending them, we would first like to know whether the interests of large institutions typically coincide with economic efficiency or oppose it. This question cannot be answered on the level of pure theory.

\section{Conclusion}

We have borrowed a mathematical model of biological evolution to formalize the idea that the law can improve by an unguided evolutionary process. Our results suggest that differential litigation (more frequent litigation of bad laws) and differential investment (more spent on preparing the case against bad laws than on defending them) will have a moderate influence on

realistic, only that it formulates a real force which affects the decision to litigate in the opposite direction from extant theories. The relative strength of the forces cannot be judged a priori; econometric evidence is needed to decide whether efficient laws are litigated less often than inefficient laws.

34 Goodman, supra note 3. Landes \& Posner, supra note 31, offer a more sophisticated model which leads to the same result: that efficient rules are litigated more often than inefficient ones. 
the persistence of good rules in a legal process without judicial insight. The law will never reach the best state and remain there forever, but these evolutionary forces will improve the law relative to what it could be without them. For very simple legal processes, the legal system will tend towards a stable state in which good laws prevail a greater proportion of time than bad laws.

We have shown the extent to which evolutionary forces can improve the law, but we have left unanswered the question whether inefficient laws are really litigated more often than efficient laws, or whether more is really invested in preparing the case against inefficient laws. In our judgment $a$ priori models, such as those derived from mathematical game theory, cannot answer this question. We cannot say whether legal evolution will have the tendencies described in our theorems because we do not know whether the forces are at work which these theorems assume. A definitive answer must come from applying econometrics to the law.

\section{APPENDIX}

We formalize the exposition of the text and provide proofs of our theorems. Consider a set of $n$ legal rules $s_{1}, s_{2}, \ldots, s_{n}$, a vector $q=\left(q_{1}, q_{2}, \ldots q_{n}\right)$ of probabilities that rule $i$ will be litigated, and a matrix $\left(p_{i j}\right)$ of probabilities that, if litigated, rule $i$ will be changed to rule $j$. We make the following assumptions:

A1: Finitude. $n$ is finite and the rules can be ordered such that rule $s_{i}$ is at least as desirable as rule $s_{j}$ if and only if $i>j$. To avoid triviality we assume that $s_{n}$ is strictly more desirable than $s_{1}$.

A2: No judicial learning. The probability that a judge will change the state of the world from $s_{i}$ to $s_{j}$ is independent of time, or for all $i$ and $j, p_{i j}$ is constant for all time. (This is the defining characteristic of a Markov process.)

A3: No judicial insight. There is a positive probability both that a judge will change any rule which is litigated and that the change can be either an improvement or worsening:
(i) for all $i, p_{i i}<1$;
(ii) for $i \neq 1$, and $i \neq n, p_{i, i-1}>0$ and $p_{i, i+1}>0$;
(iii) $p_{12}>0$ and $p_{n, n-1}>0$;
(iv) for some $i, p_{i i} \neq 0$.

A4: Complete litigation. Every state $i$ is litigated with positive probability, or $q_{i}>$ 0 for all $i$.

Assumptions A1-A4 lead to a transition matrix that is the object of study:

$$
\left[\begin{array}{llll}
a_{11} & a_{12} & \ldots & a_{1 n} \\
a_{21} & a_{22} & \ldots & a_{2 n} \\
a_{n 1} & a_{n 2} & \ldots & a_{n n}
\end{array}\right]=\left[\begin{array}{ccccc}
1-q_{1} & 0 & 0 & \ldots & 0 \\
0 & 1-q_{2} & 0 & \ldots & 0 \\
0 & & & & \\
0 & 0 & 1-q_{n}
\end{array}\right]
$$




$$
+\left[\begin{array}{cccc} 
& & & \\
q_{1} 0 & \ldots & 0 \\
0 & q_{2} & \ldots & 0 \\
& & & \\
0 & 0 & \ldots & q_{n}
\end{array}\right]\left[\begin{array}{lll} 
& \\
p_{11} p_{12} & \cdots & p_{1 n} \\
p_{21} p_{22} & \cdots & p_{2 n} \\
& & \\
p_{n 1} p_{n 2} & \cdots & p_{n n}
\end{array}\right]
$$

TheOREM 1. Assume A1-A4. Then there is a unique stable state in which the expected proportion of time that each rule prevails is strictly positive.

Proof. This is an application of the following theorem in Breiman. ${ }^{35}$ If there are only a finite number of states and all pairs of states $i, j$ communicate (can be reached in finite time with positive probability), then (a) all states are regularly recurrent and (b) if the motion is aperiodic, then the system is stable. By Al the number of states is finite. By A2 the system is a Markov process. Assume that $i$ and $j$ do not communicate. For convenience let $j=i+k$ and let there be no finite path from $i$ to $j$ with positive probability. But we can travel from $i$ to $j$ in $k$ periods of time with probability $a_{i, i+1} X a_{i, i+2} X a_{i, i+3} \ldots X a_{i, i+k}$, and this probability is positive by $\mathrm{A} 3$ and A4, which is a contradiction. So all pairs of states communicate; by Breiman's Theorem it follows that all states are regularly recurrent. By A3 the process is aperiodic since $a_{i i}$ $\neq 0$. Stability is defined as the existence of a stable state. Uniqueness follows from Breiman's Theorem 6.27. ${ }^{36}$

Comment. Assumption A3 is overly restrictive; it can be replaced by more general and less intuitive assumptions guaranteeing indecomposability and aperiodicity of the transition matrix.

Replace A4 with a stronger assumption:

A4': Partial litigation. Every state but the most efficient one $n$ is litigated with positive probability or

$$
\begin{aligned}
& q_{i}>0 \\
& q_{n}=0
\end{aligned}
$$

Theorem 2. Assume A1-A3, $A 4^{\prime}$; then there is a unique stable state in which state $n$ prevails 100 percent of the time.

Proof. Here we rely on Breiman's Theorem 6.2637: for any two states $i$ and $j$, if the transition $i$ to $j$ is possible and if $i$ is recurrent, then $j$ is recurrent and both the transition $i$ to $j$ and $j$ to $i$ are certain.

We proceed by contradiction. Assume that state $n-1$ is recurrent; then since $q_{n-1} p_{n-1, n} \neq 0$, by the above-stated theorem, $a_{n, n-1} \neq 0$. But this contradicts A4' since $q_{n}=0$ implies $q_{n} p_{n, n-1}=a_{n, n-1}=0$. Therefore state $n-1$ is transient. Now continue by induction from states $n-2$ through 1 . If $n-k$ is recurrent, A3 and A4' imply that $n-k+1$ is recurrent which would imply that $n-1$ is recurrent. Hence all states $i<n$ are transient. Clearly state $n$ is regularly recurrent with period 1. By definition a system with a regularly recurrent state has a stable state in which that

\footnotetext{
35 Breiman, supra note 17 , at 187.

${ }^{36} I d$. at 180 .

${ }^{37} I d$. at 178 .
} 
state prevails a positive proportion of time, and by definition the transient states prevail zero proportion of time in the stable state.

We formalize the notions used in Theorem 3 . We shall say that a probability vector $x \in R^{n}$ is at least as preferred as probability vector $y$ if, and only if, for every $i$ the cumulative distribution of $x$ through state $i$ is less than or equal to the corresponding cumulative distribution of $y$. In other words, $x$ can be obtained by shifting density in $y$ to the right. We have

DEFINITION 1. For two probability vectors $x$ and $y$ in $R^{n}, x R y \Leftrightarrow$ for each $i$

$$
\sum_{j=1}^{i} x_{j} \leq \sum_{j=1}^{i} y_{j} .
$$

Comment. Not all probability vectors are comparable. For example, if $x=(.25$, $.25, .5)$ and $y=(.125, .5, .375)$, then neither $x R y$ nor $y R x$.

Assume that there are two legal processes, each of which satisfies A1-A4; the two processes can thus be described by Markov matrices denoted $A$ and $B$. These matrices have unique stable states as proved in Theorem 1 . We can prove the following proposition:

TheOREM 3. If $A$ and $B$ are Markov matrices with unique stable states $y$ and $x$ respectively, and if (i) $b_{i} R a_{i}$ for each row $i$, and (ii) $b_{n} R b_{n-1} R \ldots R b_{2} R b_{1}$, then $x R y .{ }^{37}$

Proof. This is simply Nelson and Winter's Theorem 4. ${ }^{38}$ The proof of the theorem rests on three facts: (1) that $A$ moves distributions further to the right than $B$ or $(y B)$ $R(y A)$ (note that by definition of $y, y A=y$ ); (2) that because $B$ satisfies (ii) $B$ preserves the ordering relation $R$ on vectors or $w z \Rightarrow(w B) R(z B)$; and (3) that for any probability vector $z, z B^{t} \rightarrow x$ as $t \rightarrow \infty$.

Comment. It is important to realize how limited this result is. Condition (ii) is very strong. For instance, consider any process of legal change $C$. Suppose we consider the process $C^{\prime}$ derived from $C$ by increasing the probability of litigating state 1 and leaving all else the same. Then since $c_{i j}=c^{\prime}{ }_{i j}$ for $i>1$ and $c^{\prime}{ }_{1 j} \geq c_{1 j}$ for $j>1$, condition (i) of Theorem 3 is met. Unless $C^{\prime}$ meets (ii), however, we cannot conclude that the stationary distribution of $C^{\prime}$ is better than the stationary distribution of $C$. We have no counterexample to the proposition that the process $C^{\prime}$ is better than the process $C$ in the sense that its steady-state distribution is preferred, but neither do we have a proof to back up the intuition that $C^{\prime}$ is better than $C$.

Comment. Condition (ii) of Theorem 3 is nonintuitive although it may be related to the assumption of differential litigation, A6 infra. Consider a process in which the probability that a judge will change rule $i$ to rule $j$ is constant; i.e., all rule changes are equiprobable. This assumption that $p_{i j}=p$ all $i, j$ and condition (ii) together imply that bad rules are litigated more often than good rules which is the definition of

\footnotetext{
38 We have drawn upon the Appendix in Richard R. Nelson \& Sidney G. Winter, Factor Price Changes and Factor Substitution in an Evolutionary Model, 6 Bell J. Econ. 482-86 (1975). In Nelson and Winter the columns sum to one and the theorems apply to left multiplication. In our model the rows sum to one and consequently one must right multiply (i.e., $x A=A$ ).
} 
differential litigation. To the extent $\mathrm{A4}^{\prime}$ says the efficient or best rules are never litigated it may be viewed as a limiting version of $\mathrm{A} 6$.

Our next theorem requires additional assumptions.

A5. The behavior of judges is characterized by

(i) Inertia: $p_{i j}=0$ if $|i-j|>1$

(ii) Symmetry or no insight: $p_{i, i+1}=p_{i, i-1}$

(iii) Stability of good laws: $p_{i i}<p_{j j}$ if $i<j$.

A6. More efficient laws are litigated more often, but all are litigated (differential litigation).

$$
\begin{array}{rlr}
1>q_{i}>0 & \text { all } i \\
q_{i}>q_{j} & \text { if } & i<j .
\end{array}
$$

We first prove a lemma that completely characterizes the stable states for the special Markov process defined by A5 and A6. For purposes of this lemma define $a_{10}$ to satisfy $a_{10}=a_{12}$.

Lemma. Assume A1-A6. Then

(i) The Markov process defined by $A$ has a unique stable state denoted by the probability distribution $x$;

(ii) $x_{i}=\frac{1}{t(i)}$, where $t(i)$ is the time expected to elapse between appearances of rule $i$;

(iii) $x_{i}=\left(\frac{a_{i-1, i-2}}{a_{i, i-1}}\right) x_{i-1} \quad$ for $\quad i>1$

(iv)

$$
x_{i}=\frac{\prod_{j \neq i} a_{j, j-1}}{\sum_{j=1}^{n}\left(\prod_{k \neq j} a_{k, k-1}\right)} .
$$

Proof. We first show that the process $A$ defined by the assumptions is indecomposable and aperiodic with all rules regularly recurrent. The regular recurrence of each rule and the indecomposability of the transition matrix $A$ follow directly from our Theorem 1 . We prove aperiodicity by contradiction.

Assume the chain has period $d>1$. Then there are $d$ disjoint sets $B_{1}, \ldots, B_{d}$ through which the chain cycles. Assume we have arranged the subsets in order so that the system moves from state $r$ to state $r+1 \bmod d$. Consider the state $i$ guaranteed by $A 6$ in which $a_{i i}>0$. It belongs to some subset $B_{r}$. When the system is in state $r$ it must on the next move go to $B_{r+1} \bmod d$. But $a_{i t} \neq 0$ implies that state $i \epsilon$ $B_{r+1} \bmod d$. This contradicts the fact that $B_{r}$ and $B_{r+1}$ are disjoint. Therefore $d=1$ and the process is aperiodic.

Statements (i) and (ii) now follow directly from Theorems 6.26 and 6.30 of Breiman. ${ }^{39}$ Theorem 6.30 states that an aperiodic, indecomposable process with all states regularly recurrent is stable. Theorem 6.26 states that a stable system of regularly recurrent states has an unique stationary initial distribution given by (ii).

39 Breiman, supra note 17 , at $178,187$. 
We now find the unique stationary distribution. That is, we solve the simultaneous equation system $x A=x$. Let us define $a_{10}$ by $a_{10}=a_{12}$. We now establish statement (iii) by induction.

For $i=2$,

$$
\begin{aligned}
x_{1}=a_{11} x_{1}+a_{21} x_{2} & =\left(\frac{a_{21}}{\left(1-a_{11}\right)}\right) x_{2} \\
& =\left(\frac{a_{21}}{a_{12}}\right) x_{2} \text { since } 1=a_{11}+a_{12} \text { by A3 } \\
& =\left(\frac{a_{21}}{a_{10}}\right) x_{2} \text { by definition of } a_{10} .
\end{aligned}
$$

Similarly for $i=3$ we have

$$
\begin{aligned}
x & =a_{12} x_{1}+a_{22} x_{2}+a_{32} x_{3} \\
& =a_{12}\left(\frac{a_{21}}{a_{10}}\right) x_{2}+a_{22} x_{2}+a_{32} x_{3} \text { by substitution for } x_{1} \\
& =\left(\frac{a_{32}}{a_{21}}\right) x_{3}
\end{aligned}
$$

because, by $\mathrm{A} 5$ and $\mathrm{A} 6, a_{21}=a_{23}=1-a_{21}-a_{22}$.

Now assume that the formula (iii) is true for all $k^{\prime}<k-1<n$. Then

$$
x_{k-1}=a_{k-2, k-1} x_{k-2}+a_{k-1, k-1} x_{k-1}+a_{k, k-1} x_{k}
$$

but, by hypothesis,

$$
x_{k-2}=\left(\frac{a_{k-1, k-2}}{a_{k-2, k-3}}\right) x_{k-1}=\left(\frac{a_{k-1, k-2}}{a_{k-2, k-1}}\right) x_{k-1},
$$

where the final equality follows from A5. Hence:

$$
\begin{aligned}
x_{k-1} & =a_{k-1, k-2} x_{k-1}+a_{k-1, k-1} x_{k-1}+a_{k, k-1} x_{k} \\
& =\left(\frac{a_{k, k-1}}{a_{k-1, k-2}}\right) x_{k}
\end{aligned}
$$

by $\mathrm{A} 5$ and $\mathrm{A} 6$.

The statement (iii) thus holds for all $\mathrm{k}<n$. For $\mathrm{k}=n$ we have

$$
\begin{aligned}
x_{n}=a_{n-1, n} x_{n-1}+a_{n n} x_{n} & =\left(\frac{a_{n-1, n}}{a_{n, n-1}}\right) x_{n-1} \\
& =\left(\frac{a_{n-1, n-2}}{a_{n, n-1}}\right) x_{n-1}
\end{aligned}
$$

by $\mathrm{A} 5$ and $\mathrm{A} 6$.

To establish statement (iv) we need only recall that

$$
1=\sum_{i=1}^{n} x_{i}
$$


By applying (iii) recursively we get

$$
\begin{aligned}
x_{i} & =\left(\frac{a_{i-1, i-2}}{a_{i, i-1}}\right) x_{i-1} \\
& =\left(\frac{a_{10}}{a_{i, i-1}}\right) x_{1},
\end{aligned}
$$

so

$$
1=\sum_{i=1}^{n}\left(\frac{a_{10}}{a_{i, i-1}}\right) x_{1}=a_{10} x_{1} \sum_{i=1}^{n} \frac{1}{a_{i, i-1}}
$$

or

$$
x_{1}=\left(\frac{1}{a_{10}}\right)\left(\begin{array}{c}
1 \\
\sum_{1}^{n} \frac{1}{a_{i, i-1}}
\end{array}\right)
$$

But

$$
\sum_{i=1}^{n} \frac{1}{a_{i, i-1}}=\frac{\left(\sum_{i=1}^{n}\left(\prod_{j \neq i} a_{j, j-1}\right)\right)}{\left(\prod_{i=1}^{n} a_{i, i-1}\right)}
$$

and

$$
x_{1}=\frac{1}{a_{10}} \frac{\left(\prod_{i=1}^{n} a_{i, i-1}\right)}{\left(\sum_{i=1}^{n}\left(\prod_{j \neq i} a_{j, j-1}\right)\right)}=\frac{\prod_{i>1} a_{i, i-1}}{\left(\sum_{i=1}^{n}\left(\prod_{j \neq i} a_{j, j-1}\right)\right)} .
$$

Thus

$$
x_{i}=\frac{\left(\prod_{j \neq i} a_{j, J-1}\right)}{\left(\sum_{i=1}^{n}\left(\prod_{k \neq i} a_{k, k-1}\right)\right)} .
$$

Q.E.D.

We now prove that if the probability of litigation for rule $j$ increases, the proportion of time which a rule $i, i \neq j$, governs increases and the rate of increase is greater for more efficient rules. We have

Theorem 4. Assume A1-A6. Then, if the probability of litigating state $j$ increases $\left(\Delta_{q_{j}}>0\right)$,

(i) $\frac{\partial x_{i}}{\partial q_{j}}>0 \quad$ i $\neq j$

(ii) $\frac{\partial x_{i}}{\partial q_{j}}>\frac{\partial x_{k}}{\partial q_{j}} i>k, i, k \neq j$ 
(iii) $x_{i}>x_{j}$, if $i>j$ (the recurrence time of more efficient states is less).

Proof. We recall that $a_{i j}=q_{i} p_{i j}$ for $i \neq j$. The theorem follows from differentiating the expression (iv) in the lemma. Thus

$$
\begin{aligned}
\frac{\partial x_{i}}{\partial q_{j}} & =\frac{\partial}{\partial q_{j}} \cdot \frac{\left(\prod_{k \neq i} a_{k, k-1}\right)}{\left(\sum_{k=1}^{n}\left(\prod_{k^{\prime} \neq k} a_{k^{\prime}, k^{\prime}-1}\right)\right)} \\
& =\frac{p_{j, j-1}\left[\left(\prod_{k \neq i, j} a_{k, k-1}\right)\left(\sum_{k=1}^{n} \prod_{k^{\prime} \neq k} a_{k^{\prime}, k^{\prime}-1}\right)-\left(\sum_{k=j} \prod_{k^{\prime} \neq k, j} a_{k^{\prime}, k^{\prime}-1}\right)\left(\prod_{k \neq i} a_{k, k-1}\right)\right]}{d^{2}} \\
& =\frac{p_{j, j-1}\left(\prod_{k \neq i, j} a_{k, k-1}\right)\left[\sum_{k=1}^{n}\left(\prod_{k^{\prime} \neq k, j} a_{k^{\prime}, k^{\prime}-1}\right)-\sum_{k \neq j}\left(\prod_{k^{\prime} \neq k, j} a_{k^{\prime}, k^{\prime}-1}\right) a_{j, j-1}\right]}{d^{2}} \\
& =\frac{p_{j, j-1}\left(\prod_{u \neq u, j} a_{u, u-1}\right)\left(\prod_{u \neq j} a_{u, u-1}\right)}{d^{2}}>0,
\end{aligned}
$$

where $\quad d=\sum_{k=1}^{n}\left(\prod_{k^{\prime} \neq k} a_{k^{\prime}, k^{\prime}-1}\right)$

This proves (i).

To prove (ii) consider $i>i^{\prime}$. Then

$$
\frac{\left(\frac{\partial x_{i}}{\partial q_{j}}\right)}{\left(\frac{\partial x_{i^{\prime}}}{\partial q_{j}}\right)}=\frac{\prod_{k \neq i, j} a_{k, k-1}}{\prod_{k \neq i^{\prime} j} a_{k, k-1}}=\frac{a_{i^{\prime}, k^{\prime}-1}}{a_{i, i-1}}
$$

It is easy to calculate that this ratio exceeds unity by using parts (ii) and (iii) of assumption A5.

The ratio of recurrence times to consecutive states is, using statement (iii) of the lemma and A5, given by

$$
\frac{x_{i}}{x_{i-1}}=\frac{a_{i-1, i-2}}{a_{i, i-1}}>1
$$

Q.E.D. 
HeinOnline -- 9 J. Legal Stud. 1641980 